\title{
Power law tails of time correlations in a mesoscopic fluid model
}

\author{
M. Ripoll ${ }^{1,2}$ and M. H. Ernst ${ }^{3,4}$ \\ ${ }^{1}$ Institut für Festkörperforschung, Forschungszentrum Jülich - D-52425 Jülich, Germany \\ ${ }^{2}$ Dpto Física Fundamental, UNED, C/Senda del Rey 9, 28040 Madrid, Spain \\ ${ }^{3}$ CNLS, Los Alamos National Laboratory, Los Alamos, New Maxico 87545, USA \\ ${ }^{4}$ Institute for Theoretical Physics, Utrecht University, Princetonplein 5, P.O. Box 80.195, 3508 TD Utrecht, The Netherlands
}

(Received 14 February 2005; published 1 July 2005)

\begin{abstract}
In a quenched mesoscopic fluid, modeling transport processes at high densities, we perform computer simulations of the single particle energy autocorrelation function $C_{e}(t)$, which is essentially a return probability. This is done to test the predictions for power law tails, obtained from mode coupling theory. We study both off and on-lattice systems in one- and two-dimensions. The predicted long time tail $\sim t^{-d / 2}$ is in excellent agreement with the results of computer simulations. We also account for finite size effects, such that smaller systems are fully covered by the present theory as well.
\end{abstract}

DOI: 10.1103/PhysRevE.72.011101

PACS number(s): 05.20.Dd, 05.40.-a, 05.10.-a

\section{INTRODUCTION}

In classical fluids in thermal equilibrium the velocity autocorrelation function $(\mathrm{VACF})$ decays algebraically $\sim t^{-d / 2}$ at large times, and so do all Green-Kubo integrands, as established by molecular dynamics [1-3], mode coupling [4,5], and kinetic theory [6]. These functions are equilibrium time correlation function $\langle J(t) J(0)\rangle_{0}$ of single- or $N$-particle currents, whose time integrals give the transport coefficients, such as self-diffusion, viscosity, or heat conductivity.

The long time tails are not restricted to current-current correlations, but apply to large classes of time correlations, such as single site correlations, provided the dynamics obeys a conservation law. Similar single particle correlations with a long time tail (LTT) exist in fluids [5]. These tails have a rather universal shape, in the sense that they are determined by the decay of the slow macroscopic modes of the system, and are independent of the microscopic details.

Recently, we have discussed in paper I [7] a simplified mesoscopic model for transport in fluids, in which the rapid short range fluctuations are averaged out. It is referred to as random DPD solid (dissipative particle dynamics), and defined by a fluctuating heat equation (Langevin equation), where the random force satisfies the fluctuation-dissipation theorem. It is a special case of general DPD fluids $[7,8]$, in which point particles are characterized by positions $\mathbf{r}_{i}$, velocities $\mathbf{v}_{i}$ and possibly by an internal energy $\epsilon_{i}(i=1,2, \ldots, N)$.

The simplification has been obtained by quenching the translational degrees of freedom of the DPD particles. The only dynamic degrees of freedom left are the internal energies $\epsilon_{i}(i=1,2, \ldots, N)$ of the particles, where the total energy $\Sigma_{i} \epsilon_{i}(t)=E$ is conserved. The only remaining transport process is heat diffusion between fixed particles, where energy hops instantaneously between interacting pairs $\{i j\}$ within the interaction range $\left(r_{i j} \leqslant r_{c}\right)$. This transport mechanism is called collisional transfer. The direction of the energy flow is determined by the "local temperature gradient" $\left(\epsilon_{j}-\epsilon_{i}\right)$, and heat conduction is only possible for densities above a percolation threshold $\rho_{p}$. In the underlying percolating structure two particles are connected (by a bond) if the distance between them satisfies, $r_{i j} \leqslant r_{c}$. So we are dealing with bond percolation diffusion on the random proximity network, where the transition rate or conductivity across a bond is constant.

In this paper we study the energy autocorrelation function of a single particle $C_{e}(t)$. It is the analog of the probability of return to the origin, $P(R=0, t)$, where $P(R, t)$ satisfies a diffusion equation.

The random DPD model is complementary to the Lorentz gas with overlapping scatterers in several respects. The latter has only kinetic transport of particles and the former has only the transport mechanism of collisional transfer of energy. In classical fluids both mechanisms are present. The former is dominant at low and moderate densities and the latter at liquid densities.

The overlapping Lorentz gas also has a percolation threshold, where diffusion only occurs below the percolation density. A more detailed comparison is given in paper I, where the similarities and differences between both models for percolation diffusion are discussed. The diffusion phenomena in both models occur on random percolation structures, which are essentially each other's duals [7]. In Lorentz gases the return probability has a LTT $\sim t^{-d / 2}$, and the VACF has a LTT $\sim t^{-1-d / 2}$. In the hydrodynamic interpretation of the LTT of the VACF in classical fluids in terms of vorticity diffusion, as given in by Alder and Wainwright, the velocity autocorrelation function of a tagged particle may also be interpreted as the return probability of the initial momentum to the tagged particle itself, where $d$ is the spatial dimension of the system [9-11]. A further property of the Lorentz gas is that the power law tail is absent when the scatterers are placed on a periodic lattice [12]. The present paper also analyzes what happens to the long time tails when the particles are put on a lattice. In fact, the interpretation of $C_{e}(t)$, being a return probability in a diffusion process, already suggests that $C_{e}(t) \sim P(R=0, t)$ should also have a power law tail $\sim t^{-d / 2}$ on a regular lattice.

As the long wavelength decay modes of classical fluids and DPD models are essentially the same, the decay of the 
corresponding equilibrium correlations should also be the same. This is in fact implied by Onsager's regression hypothesis [13]. For the VACF in DPD fluids [14] and for the energy autocorrelation function (EACF) in a DPD solid [15] more specialized mode coupling arguments have been developed to show the existence of a power law tail $\sim t^{-d / 2}$. Unfortunately the predictions, in particular the one for the DPD solid, could not be confirmed by the existing computer simulations of three-dimensional DPD systems, because the systems studied were too small, e.g., $N=1000, L / r_{c}=5$ and $N=2000, L / r_{c}=6$, where $V=L^{d}[15]$. Consequently, the short time kinetic decay, $\exp \left[-\omega_{0} t\right]$, and the slow decay of the macroscopic diffusive modes, $\exp \left[-k^{2} D t\right]$, were equally fast (no separation of time scales), and power law tails in the EACF could not be observed because of strong finite size effects. The system sizes in our present computer simulations of the two-dimensional DPD solid are sufficiently large to observe the power law tails.

The plan of the paper is as follows. In Sec. II the definitions and results for the DPD solid are briefly summarized insofar as needed in the present paper. In Sec. III the mode coupling theory of Refs. $[4,5]$ is applied to obtain the explicit form of the LTT of $C_{e}(t)$ in the random DPD solid. In Sec. IV the results are compared with computer simulations of the two-dimensional case. In Sec. IV it is also explained how the same results for the time correlations can be obtained from deterministic simulations, where the dynamics is free of statistical noise. In Sec. V the LTT's of time correlation functions are studied on a lattice. Conclusions are presented in Sec. VI.

\section{RANDOM DPD SOLID}

The system consists of $N=n V$ point particles at fixed random positions $\mathbf{r}_{i}(i=1,2, \ldots, N)$, contained in a volume $V$ $=L^{d}$, and obeying periodic boundary conditions. Each DPD particle interacts with all particles inside its interaction sphere of radius $r_{c}$. The only dynamical variables in the system are the internal energies $\epsilon_{i}$ of the particles, and the time evolution is given by the Langevin equation

$$
\frac{d \epsilon_{i}}{d t}=\lambda_{0} \sum_{j}{ }^{\prime} w\left(r_{i j}\right)\left(\epsilon_{j}-\epsilon_{i}\right)+\sum_{j}{ }^{\prime} \tilde{F}_{i j}(t) .
$$

Here $w(r)$ is a positive interaction function of finite range $r_{c}$, normalized as $\int d \mathbf{r} w(r)=r_{c}^{d}$. In the present paper $w(r)=$ const $\theta\left(r_{c}-r\right)$ is proportional to the unit step function, which vanishes for $r>r_{c}$. The kinetic rate constant $\lambda_{0}$ is a model parameter that determines the interaction frequency. The prime on the summation sign indicates that $j \neq i$. Here $\widetilde{F}_{i j}=-\widetilde{F}_{j i}$ is Gaussian white noise, whose explicit form is given in paper I. It satisfies the detailed balance conditions, guaranteeing that the system reaches thermal equilibrium.

To discuss the short time decay of an energy fluctuation we consider

$$
\nu=\sum_{j}{ }^{\prime}\left\langle\theta\left(r_{c}-r_{i j}\right)\right\rangle \equiv \rho \varpi_{d},
$$

where $\nu$ is the mean number of $j$ particles inside $r_{i j} \leqslant r_{c}$ that interact with the $i$ th particle. Moreover, $\varpi_{d}=\pi^{d / 2}$ /
$\Gamma(1+d / 2)$ is the volume of a $d$-dimensional unit sphere $(d=1,2, \ldots)$, and $\rho=n r_{c}^{d}$ is the reduced density.

The average $\langle\cdots\rangle$ denotes a quenched average over the random configurations of the fixed DPD particles. The basic relaxation rate at short times can be estimated from Eqs. (1) and (2) as

$$
\omega_{0}=\lambda_{0} \sum_{j}{ }^{\prime}\left\langle w\left(r_{i j}\right)\right\rangle=\rho \lambda_{0} .
$$

Each DPD particle is a mesoscopic subsystem with a density of internal states $\sim \epsilon^{\alpha}$, where $\alpha$ is proportional to the number of internal degrees of freedom of a DPD particle, and satisfies $\alpha \gg 1$. In thermal equilibrium the single particle distribution function is

$$
\psi_{0}(\epsilon)=\frac{\beta}{\Gamma(\alpha+1)}(\beta \epsilon)^{\alpha} \exp [-\beta \epsilon]
$$

where $T=1 / k_{B} \beta$ is the temperature. Also note that the evolution equation conserves the total energy, i.e., $\sum_{i} \epsilon_{i}(t)=E$ $=$ const, as both terms in Eq. (1) are antisymmetric in $i$ and $j$.

A final comment regarding the stochastic differential equation (1), which contains multiplicative noise: As discussed in paper I, the difference between the Îto and Stratonovich interpretation of the Langevin equation and the corresponding Fokker Planck equation can be neglected to leading order in $\mathcal{O}(1 / \alpha)$ in the present model.

In this paper we study the long time tail of the energy autocorrelation function $(\mathrm{EACF})$ in thermal equilibrium,

$$
C_{e}(t)=\frac{1}{N} \sum_{i}\left\langle\delta \epsilon_{i}(t) \delta \epsilon_{i}(0)\right\rangle_{0},
$$

where $\langle\cdots\rangle_{0}$ is an average over the canonical distribution $\Pi_{j} \psi\left(\epsilon_{j}\right)$. Here $\delta \epsilon_{j}(t)=\epsilon_{j}(t)-\left\langle\epsilon_{j}\right\rangle_{0}$ is the energy fluctuation with $\left\langle\epsilon_{j}\right\rangle_{0} \simeq \alpha / \beta$. It is essentially the return probability of the initial energy $\delta \epsilon_{i}(0)$ to the particle, from which it originated. It is the analog of the velocity autocorrelation function, $C_{v}(t)=\left\langle v_{x}(t) v_{x}(0)\right\rangle_{0}$ in the same sense as the VACF is the return probability of the initial momentum. The latter picture explains its LTT $\sim t^{-d / 2}[1]$. However, the time integral of the VACF equals the coefficient of self-diffusion $D$, whereas the time integral of the EACF is not related to the heat diffusivity $D_{T}$, or to any other transport coefficient.

The explicit form of the power law tail of the EACF has been given in Refs. $[5,15]$. The derivation can essentially be copied from the corresponding derivation for the VACF in fluids [4]. We only give an outline. We first express $\delta \epsilon_{1}(t)=\int d \mathbf{r} \delta e_{s}(r, t)$ where $\delta e_{s}(r, t)=\delta \epsilon_{1}(t) \delta\left(\mathbf{r}-\mathbf{r}_{1}\right)$ is the local energy density of tagged particles. According to the Onsager regression hypothesis, the average decay of fluctuations around thermal equilibrium follows the macroscopic approach to equilibrium. This implies that a local fluctuation $\delta e_{s}$ rapidly decays to its value in local equilibrium, i.e., 


$$
\delta \epsilon_{1}(t)=\int d \mathbf{r} \delta e_{s}(r, t) \simeq \int d \mathbf{r} n_{s}(r) \mathcal{C} \delta T(r, t) \simeq \frac{\mathcal{C}}{V} \sum_{\mathbf{k}} \delta T_{\mathbf{k}}(t) n_{-\mathbf{k}}^{s}
$$

where $\mathcal{C}=\alpha k_{B}$ is the specific heat per DPD particle, and $\delta T(\mathbf{r}, t)$ is the local temperature fluctuation, $n_{s}(\mathbf{r})=\delta\left(\mathbf{r}-\mathbf{r}_{1}\right)$ the quenched local density of tagged particles, and $a_{\mathbf{k}}$ denotes the Fourier transform of $a(\mathbf{r})$. The subsequent slow evolution is controlled by the heat mode $\delta T_{\mathbf{k}}(t)=\delta T_{\mathbf{k}}(0) \exp \left[-k^{2} D_{T} t\right]$. The tagged particle density $n_{\mathbf{k}}^{s}$ is a static mode. Inserting $\delta T_{k}$ in Eqs. (5) and (6), and using the relations $n_{k}^{s} \rightarrow 1$ as $k \rightarrow 0$, and $\delta T_{k}(0) \rightarrow \delta \epsilon_{1}(0) / \mathcal{C}$ as $k \rightarrow 0$, we obtain in the long time limit

$$
C_{e}(t)=\frac{1}{n V} \sum_{\mathbf{k}} \exp \left[-k^{2} D_{T} t\right] C_{e}(0)
$$

This yields in the thermodynamic limit as $N=n V \rightarrow \infty$ at fixed $n$, the power law tail,

$$
\begin{aligned}
\frac{C_{e}(t)}{C_{e}(0)} & \rightarrow \frac{1}{n} \int \frac{d \mathbf{k}}{(2 \pi)^{d}} \exp \left[-k^{2} D_{T} t\right] \\
& =\frac{1}{n\left(4 \pi D_{T} t\right)^{d / 2}} \\
& =\frac{1}{\rho\left(4 \pi t^{*}\right)^{d / 2}}\left(t^{*} \gg 1\right)
\end{aligned}
$$

In the first step we used the relation $C_{e}(0)=\left\langle(\delta \epsilon)^{2}\right\rangle_{0} \simeq \alpha / \beta^{2}$, and in the last we introduced the dimensionless time $t^{*}=D_{T} t / r_{c}^{2}$, where $t_{D}=r_{c}^{2} / D_{T}(\rho)$ is the characteristic time for heat diffusion. The integral representation (8), $\int d \mathbf{k} P_{\mathbf{k}}(t) \sim P(R=0, t)=\left(4 \pi D_{T} t\right)^{-d / 2}$, of the long time limit of $C_{e}(t)$ shows that $P(R, t)$ is the solution of the heat diffusion equation, and that $P(R=0, t)$ is a return probability.

The main goal of this paper is to test the theoretical prediction about the long time tail (LTT) in $C_{e}(t)$.

\section{LONG TIME TAILS IN STOCHASTIC AND DETERMINISTIC SIMULATIONS}

The goal of this section is to compare the predictions (7) for the long time tails with the results of computer simulations in the two-dimensional random solid described by the Langevin equation (1). The values of the heat diffusivity, to be used in the comparison, have been obtained from computer simulations, using the methods of paper I, and agree well with the mean field result $D_{\infty}(\rho)=\lambda_{0} \rho r_{c}^{2} /[2(d+2)]$ at high densities. As $\rho$ decreases, $D_{T}(\rho)$ decreases faster than linear, and vanishes at a nonvanishing threshold density $\rho_{p}$, which coincides with the percolation threshold for continuum percolation of overlapping spheres. In two dimensions it has the value $\rho_{p} \simeq 1.43629$ [16], and in three dimensions one has $\rho_{p}=0.65296$ [17].

To measure LTT's of equilibrium time correlation functions the DPD solid has to be in a state of thermal equilibrium. This is achieved by initializing the system in a state with a uniform energy distribution $\epsilon_{i}(0)=E / N$, where $E$ is

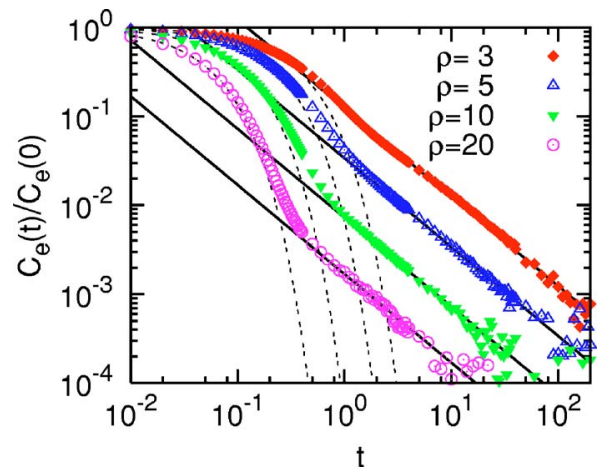

FIG. 1. (Color online) Simulation data for EACF, $C_{e}(t)$ vs $t$ at different reduced densities $\rho$ [defined below Eq. (2)] in the twodimensional random solid for a system of $N=\rho\left(L / r_{c}\right)^{2}=5 \times 10^{4}$ particles, compared with theoretical predictions: the dashed lines represent the short time exponential decay, and the solid lines the algebraic LTT $\sim 1 / t$. The higher the density the sooner the LTT is reached.

the total energy. In principle the Langevin forces, which satisfy the detailed balance conditions, drive the system to a state of thermal equilibrium, described by $\psi_{0}$ in Eq. (4). This is only the case in a pure ergodic system. However, with decreasing density larger fractions of particles are contained in small disconnected islands, which have no interactions with the bulk of the particles in the system, i.e., belong to small independent ergodic subsystems, and cannot redistribute their initial energies over the bulk system. Hence initialization of the system in the canonical equilibrium state can only be guaranteed if the fraction of island particles is negligible, i.e., for $\rho \gg \rho_{p}$.

Figures 1-3 show the simulation data for the energy autocorrelation function $C_{e}(t)$ of a DPD particle in a twodimensional system of $N=\rho\left(L / r_{c}\right)^{2}$ particles at different densities. There is excellent agreement of computer simulations with theoretical predictions. In the simulations we measure length in units of $r_{c}$, and times in units of $1 / \lambda_{0}$. At short times the decay is exponential, $\exp \left[-\omega_{0} t\right]$, with a rate constant $\omega_{0}=\rho \lambda_{0}$, as given in Eq. (3), in very good agreement with the simulation data. At large times the plots show the long time tail $\sim t^{-d / 2}$.

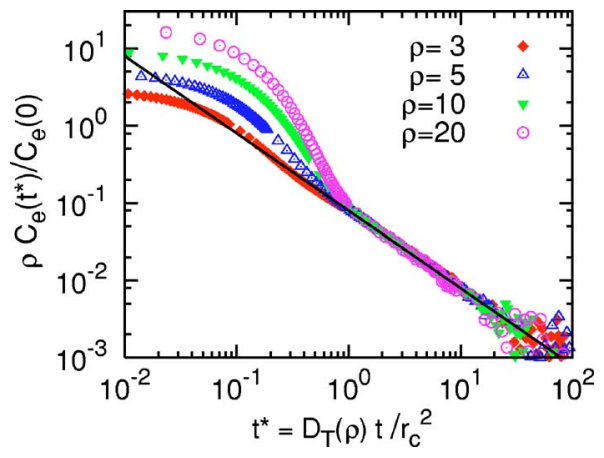

FIG. 2. (Color online) Collapse plot of the LTT's in Fig. 1 obtained by plotting $\rho C_{e}(t) / C_{e}(0)$ in Eq. (5) vs $t^{*}$, compared with the predicted LTT (solid line). Note that the crossover from exponential to algebraic decay is at $t_{D}=r_{c}^{2} / D_{T}(\rho)$. 


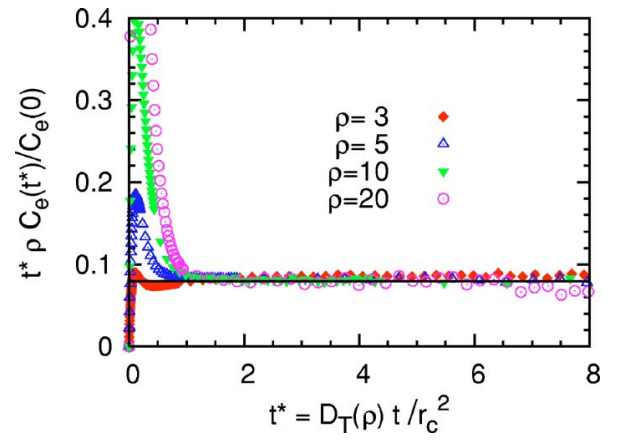

FIG. 3. (Color online) A more sensitive comparison of the collapse plot with the theoretical prediction (solid horizontal line), focusing on relatively short times.

By plotting the simulation results as $\rho C_{e}(t) / C_{e}(0)$ versus the dimensionless time $t^{*}=D_{T}(\rho) t / r_{c}^{2}$ the data for different $\rho$ can be collapsed on a single LTT curve. Moreover, the combination of Figs. 1-3 shows that the crossover time from exponential short time decay $\sim \exp \left[-\omega_{0} t\right]$ to power law decay is for all densities given by $t_{D}=r_{c}^{2} / D_{T}(\rho)$.

The finite size effects on the dynamics are controlled by the ratio $L / r_{c}=(N / \rho)^{1 / d}$. As DPD particles in absence of conservative forces are point particles, the density can become arbitrarily high. So, as $\rho$ increases at fixed $N$ the finite size effects increase. Consider the data in Figs. $1-3$ at $(\rho=5$, $L=100$ ), corresponding to $N=5 \times 10^{4}$ particles. If the number of particles is reduced to $N=10^{4}$ a faster decay than $1 / t$ decay becomes noticeable, which is statistically significant. For $N=10^{3}$ the decay is even faster, and looks exponential. It is caused by finite size effects. We return to this point later on.

The stochastic simulations above were described by the Langevin equation (1). The subsequent deterministic simulations of this section correspond to the same equation with the Langevin forces switched off $\left[\widetilde{F}_{i j}(t)=0\right]$. Moreover, the stochastic forces have no effect on the decay of the equilibrium time correlation functions $\langle\delta a(t) \delta a(0)\rangle_{0}$, provided both types of correlation functions are at $t=0$ in thermal equilibrium. This observation follows from Onsager's regression hypothesis on the average decay of fluctuations. The purpose of driving the system by Gaussian white noise, that satisfies the detailed balance conditions, is to maintain the system in thermal equilibrium, but does not affect the decay of these functions.

The above observations offer the interesting possibility to measure the LTT of equilibrium time correlations deterministically, i.e., the dynamics is free of statistical noise, provided the system is prepared initially in the proper thermal equilibrium state. Here the thermal fluctuations are only accounted for in the initial distribution. This method has also been used in Ref. [18] where the kinetic part of the stressstress correlation was measured using the lattice Boltzmann equation.

Next we discuss the results of the deterministic simulations of $C_{e}(t)$ in the two-dimensional random DPD solid at density $\rho=5$, where on average $\pi \rho$ particles are surrounding the central particle in the interaction sphere. In order to simu-

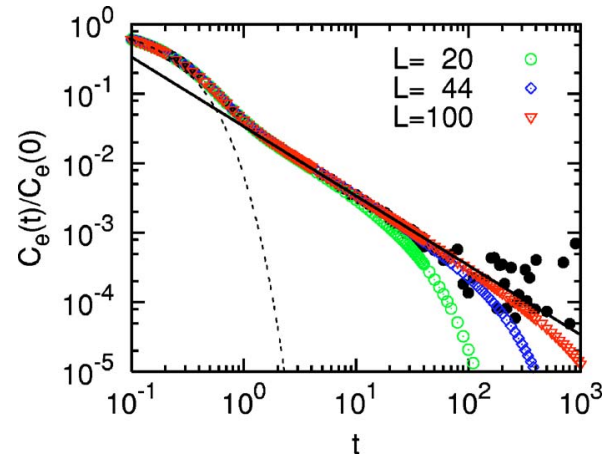

FIG. 4. (Color online) The log-log plot shows the EACF vs $t$ in the deterministic simulations at $\rho=5$ for $L / r_{c}=20,40,100$, corresponding to $N=2 \times 10^{3}, 8 \times 10^{3}, 5 \times 10^{4}$ particles. The scattered black circles $\left(L / r_{c}=44\right)$ show stochastic simulations for comparison. The straight solid line shows the predicted power law tail (8), and the dashed line indicates the short time exponential decay.

late the equilibrium time correlation function $C_{e}(t)$, we prepare the system in the initial state, described by the $N$-particle distribution, $\Pi_{i=1}^{N}\left[\psi_{0}\left(\epsilon_{i}\right) / V\right]$, and average over different runs with independent initial configurations. In spite of the additional average over different runs the computational effort for the deterministic simulations is considerably smaller than for the stochastic ones. Figures 4 and 5 show $\log$-log plots of $C_{e}(t) / C_{e}(0)$ vs $t$ at different system sizes $L / r_{c}$ with $N=\rho\left(L / r_{c}\right)^{d}$ particles. Figure 4 is focusing on the short time behavior $\exp \left[-\lambda_{0} \rho t\right]$ and power law tails $(1 / \rho)$ $\times\left[4 \pi D_{T}(\rho) t / r_{c}^{2}\right]^{-d / 2}$, and Fig. 5 on the power law tail and the ultimate exponential decay. Both figures also show for comparison the stochastic simulation at $L / r_{c}=44$ as scattered black circles. This confirms that the exponential short time and the intermediate power law behavior of the deterministic and stochastic simulations are the same, and agree with the theoretical prediction, until the time where the statistical uncertainty in the stochastic simulations has become too large. This behavior beautifully confirms Onsager's regression hypothesis.

Figure 6 shows that the ultimate decay is again exponential. This is a finite size effect, which is in excellent agree-

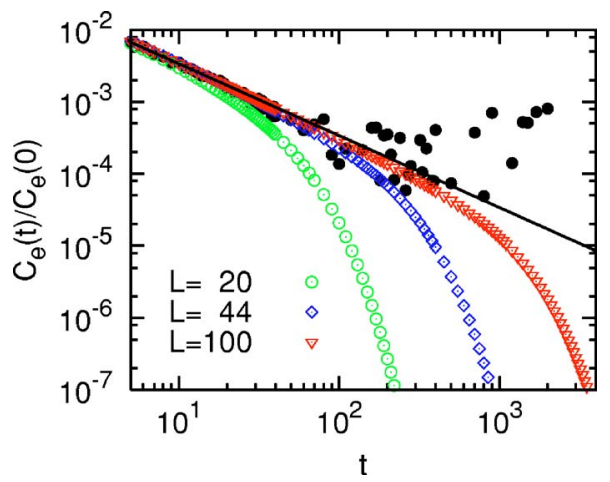

FIG. 5. (Color online) The log-log plot shows the same deterministic simulations as in Fig. 4, but focuses on the intermediate algebraic tail. The straight line is the LTT in the thermodynamic limit. The ultimate long time decay is again exponential. For the scattered black circles see the caption of Fig. 4. 


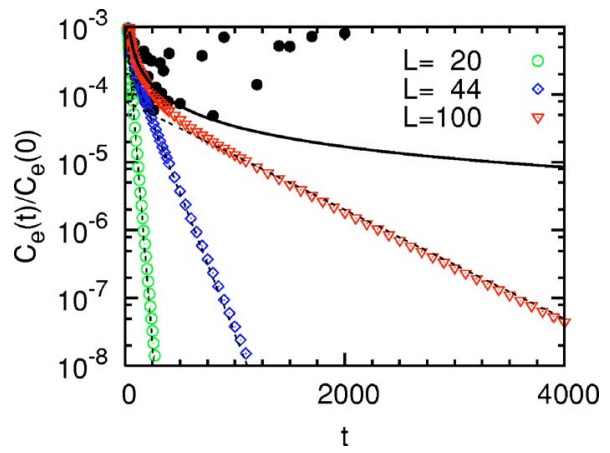

FIG. 6. (Color online) The half logarithmic plot focuses on the ultimate exponential decay in the simulations of Figs. 4 and 5, which are finite size effects, and are quantitatively accounted for by the first term in Eq. (9), $(4 / N) \exp \left[-(2 \pi / L)^{2} D_{T} t\right]$. The solid line at the top right is again the algebraic LTT for thermodynamically large systems. For the scattered black circles, see the caption of Fig. 4.

ment with the mode coupling prediction (7) for finite systems, where the $\mathbf{k}$ summation cannot be replaced by a $\mathbf{k}$ integral. On a square lattice with periodic boundary conditions $k_{\alpha}=2 \pi n_{\alpha} / L$ with $\alpha=x, y$ and $n_{\alpha}=0, \pm 1, \pm 2, \ldots$. The integral approximation to Eq. (7) is only valid in the limit of large $t$, and small $k$, such that $k^{2} t=$ const. This asymptotic approximation is appropriate as long as $D_{T} k_{\min }^{2} t \ll 1$, and breaks down at $t_{\min }$ for, say, $D_{T} k_{\text {min }}^{2} t_{\text {min }} \approx 1 / 3$, where the minimum $k$ value is $k_{\min }=2 \pi / L$. The first few terms in the finite size mode coupling formula on a square lattice follow from Eq. (7) in the form

$$
\begin{aligned}
\frac{C_{e}(t)}{C_{e}(0)} & =\frac{1}{N} \sum_{\mathbf{n}} \exp \left[-|\mathbf{n}|^{2} s\right] \\
& =\frac{1}{N}\left(4 e^{-s}+4 e^{-2 s}+4 e^{-4 s}+8 e^{-5 s}+\cdots\right),
\end{aligned}
$$

where $s=k_{\min }^{2} D_{T} t=4 \pi^{2} D_{T} t / L^{2}$, and $\mathbf{n}=\left(n_{x}, n_{y}\right)$. In Fig. 6 the straight lines of simulation data are well represented through the first term $(4 / N) e^{-s}$ of the right hand side of Eq. (9).

Furthermore, our method is not suitable to study equilibrium time correlations close to the percolation threshold, because the equilibration time $t_{0}$ diverges as $\rho \downarrow \rho_{p}$ on the percolating cluster. Moreover the finite fraction of particles contained in smaller disconnected islands form uncoupled ergodic subsystems. This prevents the system from reaching the canonical equilibrium state $\Pi_{j} \psi_{0}\left(\epsilon_{j}\right)$.

\section{DPD SOLID ON A LATTICE}

In this section we introduce a lattice version of the DPD solid in Eq. (1), where $N$ particles fill the $N$ sites of a hypercubic lattice with lattice distance $a$, and volume $V=L^{d}$ $=N a^{d}$. In this model the interaction range $r_{c}$ is the control parameter and heat diffusion only occurs for $r_{c} \geqslant a$. We will observe that the LTT of $C_{e}(t)$ in the random DPD solid survives when the DPD particles are put on a periodic lattice, as is expected for a return probability. To do so we consider the special case of the DPD solid on a lattice, where $C_{e}(t)$ can be

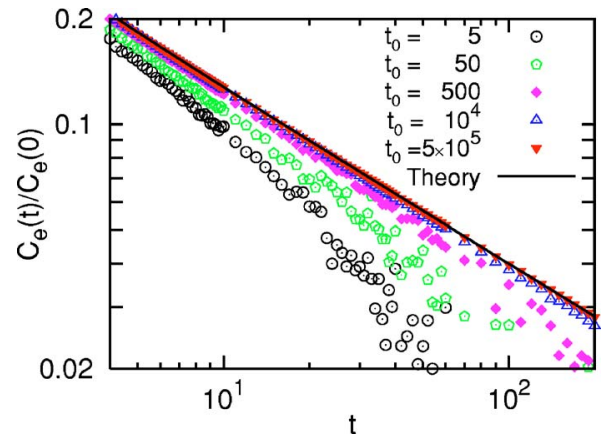

FIG. 7. (Color online) The plot shows the simulated EACF on a one-dimensional lattice with nearest neighbor interactions after different equilibration times $t_{0}=5,50,500,10^{4}$ and $5 \times 10^{5}$, as well as the exact solution (11) for the one-dimensional lattice model.

calculated exactly [5]. However, in periodic Lorentz gases the power law tail disappears in the VACF, as shown in Ref. [12].

Consider the evolution equation for the lattice space-time correlation function $C(\mathbf{n}, t)=\left\langle\delta \epsilon_{\mathbf{n}}(t) \delta \epsilon_{\mathbf{0}}(0)\right\rangle_{0}$ in thermal equilibrium, where $\mathbf{n}=\left(n_{x}, n_{y}, \ldots, n_{d}\right)$ labels the sites of a hypercubic lattice with periodic boundary conditions, and $n_{\alpha}=\{0,1,2, \ldots, L-1\}$ with $\alpha=\{x, y, \ldots, d\}$. The evolution equations are obtained by replacing $\epsilon_{i}$ in Eq. (1) with $\delta \epsilon_{\mathbf{n}}(t)$, multiplying that equation with $\delta \epsilon_{0}(0)$, and averaging over the $N$-particle equilibrium distribution function. As $\delta \epsilon_{0}(0)$ is uncorrelated with the Langevin force, the equations of motion of the lattice space-time correlation functions follow as:

$$
d C(\mathbf{n}, t) / d t=\lambda_{0} \sum_{\mathbf{m}} w\left(r_{\mathbf{n m}}\right)[C(\mathbf{m}, t)-C(\mathbf{n}, t)]
$$

with initial condition $C(\mathbf{n}, 0)=\delta_{\mathbf{n} 0}\left\langle(\delta \epsilon)^{2}\right\rangle_{0} \simeq \delta_{\mathbf{n} 0} \alpha / \beta^{2}$. This is a discrete diffusion equation, which can be solved exactly for general dimensionality [5], and the single site correlation or return probability $C(\mathbf{n}=\mathbf{0}, t)=C_{e}(t)$. For $w\left(r_{\mathbf{n m}}\right)$ restricted to nearest neighbor interactions $\left(r_{c}=a\right)$, the solution is given by [5]

$$
C_{e}(t) / C_{e}(0)=\left[e^{-\tau} I_{0}(\tau)\right]^{d} \rightarrow(2 \pi \tau)^{-d / 2} .
$$

Here $\rightarrow$ denotes the long time behavior for $\tau \gg 1$. Moreover, $I_{l}(\tau)$ with $l=0$ is a modified Bessel function with $\tau=2 D_{T} t / a^{2}$, and the heat diffusivity, $D_{T}=\lambda_{0} a^{2} / \varpi_{d}$, where units of time are consistent with Eqs. (1) and (3).

In the lattice model both characteristic time scales, the short kinetic time scale, $1 / \omega_{0}=1 / \rho \lambda_{0}$, and the long diffusive time scale $t_{D}=r_{c}^{2} / D_{T}(\rho)$, are roughly inversely proportional to the mean number of $j$ particles inside the interaction sphere $\left(r_{i j} \leqslant r_{c}\right)$ of the central particle $i$, and differ roughly by an order of magnitude in size. As $\rho \downarrow \rho_{p}$ this number decreases rapidly, and the equilibration time $t_{0}$ at $\rho_{p}$ diverges, as the interactions become weaker and weaker.

For the exactly solved case of the lattice DPD solid in $d$ dimensions with nearest neighbor interactions, Figs. 7 and 8 show for the one-dimensional case that a very long equilibration time $\lambda_{0} t_{0} \simeq 5 \times 10^{5}$ is required. After that period the exact solution $C_{e}(t)$ can be observed with its full $1 / \sqrt{t}$ tail 


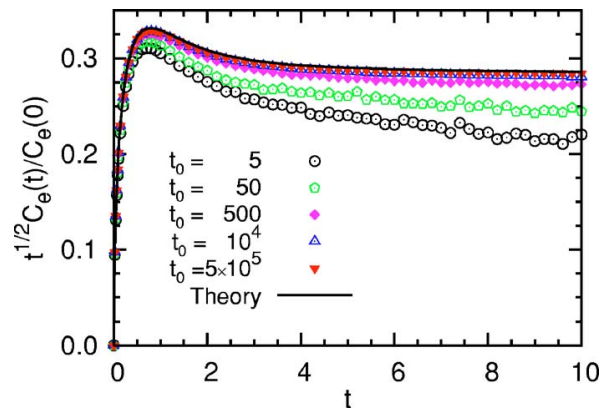

FIG. 8. (Color online) The plot shows the same data for $C_{e}(t)$ as in Fig. 7, but focuses on short times. Note that the interval around $\lambda_{0} t \simeq 1$ also equilibrates very slowly.

over the whole time interval $\lambda_{0} t \leqslant 1000$. If the equilibration time is too short, the observed LTT falls off faster than $1 / \sqrt{t}$.

For general interaction ranges $\left(r_{c}=\mathcal{N} a\right)$, where $\mathcal{N}$ represents the number of interacting lattice sites on periodic lattices, the mode couplings arguments of Refs. [5,15] produce the LTT in Eq. (8) which yields in one dimension an algebraic LTT $\sim 1 / \sqrt{t}$. In the one-dimensional lattice case it is straightforward to calculate the coefficient of heat diffusivity $D_{T}(\rho)$ analytically, as shown in the Appendix, and the results of the LTT's in the stochastic simulations are show in Figs. 9-11, which can be extended directly to higher dimensional lattices. The simulation results are in very good agreement with the predictions of the mode coupling theory.

We also note that the random DPD model in one dimension is in the thermodynamic limit nonconducting because at any given density $\rho$ there will always be a non-vanishing probability to find a single pair $(i j)$ of nearest neighbors with $r_{i j}>r_{c}$.

\section{CONCLUSIONS AND OPEN PROBLEMS}

The general conclusion from the previous sections is that the predictions of mode coupling theory for classical fluids [4] regarding the existence of LTT $\sim t^{-d / 2}$ in time correlations are in excellent agreement with the results of computer simu-

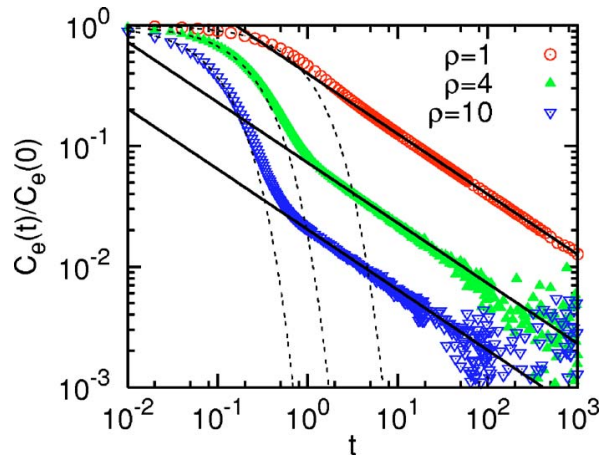

FIG. 9. (Color online) The EACF $C_{e}(t)$ in the one-dimensional lattice model at different $\rho$ for a system of $N=10^{4}$ particles compared with the theoretical predictions: the dashed line is the short time exponential decay, and the straight lines are the algebraic LTT $\sim 1 / \sqrt{t}$ (compare with Fig. 1).

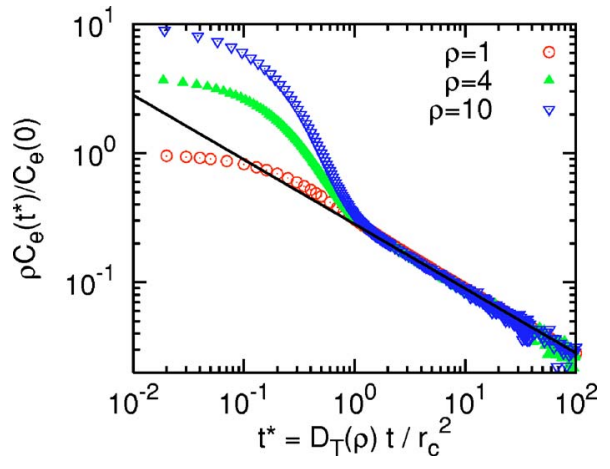

FIG. 10. (Color online) Collapse plot of the LTT in the onedimensional lattice model (compare with Fig. 2).

lations, when applied to the energy autocorrelation function $C_{e}(t)=\left\langle\delta \epsilon_{i}(t) \delta \epsilon_{i}(0)\right\rangle_{0}$ in the random DPD solid and in the lattice DPD models (with a uniform density distribution) both in one and two dimensions. Here the energy autocorrelation function $C_{e}(t)$ at large time is essentially a return probability in the same sense as the velocity autocorrelation function is in a classical fluid.

Nevertheless, a number of interesting questions about consistency of the theory remains for the LTT predictions $C_{e}(t) \propto\left(D_{T} t\right)^{-d / 2}$ for $d=1,2, \ldots$. For classical fluids in two dimensions the Navier Stokes transport coefficients do not exist. For example, let $C(t)=\left\langle v_{x}(t) v_{x}(0)\right\rangle_{0}$ with a LTT $\sim t^{-d / 2}$, be the velocity autocorrelation function. Then the long time limit of $D(t) \equiv \int_{0}^{t} d \tau C(\tau)$ yields the coefficient of self diffusion $D$, if the integral exists. However, this relation would lead to the result $D(t) \sim\{\sqrt{t}, \ln t\}$ for $d=\{1,2\}$, respectively, in the long time limit, leading to the nonexistence of the Navier-Stokes transport coefficients in one-dimensional and two-dimensional fluids. Then, self-consistent mode coupling theory, ring kinetic theory, and other renormalization procedures [19] lead to a "renormalized super-long time tail" of the form $[19,20]$

$$
\begin{gathered}
D(t) \sim\left\{t^{1 / 3}, \sqrt{\ln t},\right. \\
C(t) \sim d D(t) / d t=\left\{t^{-2 / 3}, 1 /[t \sqrt{\ln t}]\right\}
\end{gathered}
$$

for $d=\{1,2\}$, respectively. In fact, van der Hoef and Frenkel [20] have confirmed for the VACF in two-dimensional fluids

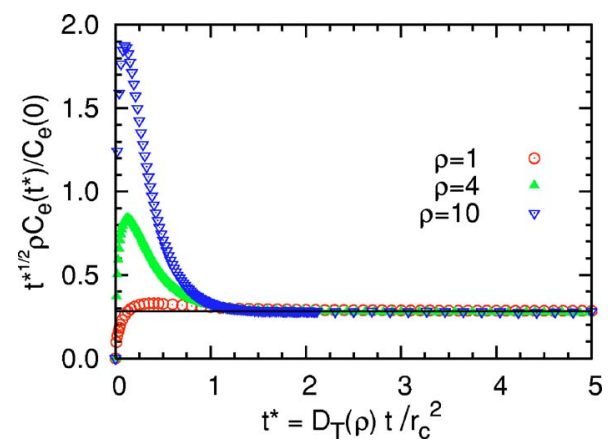

FIG. 11. A more sensitive comparison of the collapse plot of Fig. 10 for short times (compare with Fig. 3) with the theoretical predictions. 
the existence of a "faster-than- $t^{-1}$ tail" of $C(t)$ by computer simulations using lattice gas cellular automata. However, our computer simulations of $C_{e}(t)$ in Figs. 1-3 and 9-11 do not show any deviations from the predictions of the simple mode coupling theory in Eq. (8), and seem to confirm the LTT $\sim t^{-d / 2}$, as well as the finiteness of $D_{T}$.

This simple behavior is further confirmed by the observations (i) that $C_{e}(t)$ in the two-dimensional random solid in Figs. 1-3 and in the one-dimensional lattice versions in Figs. 9-11 behave essentially the same, and (ii) that the lattice models contain as a special case the model with nearest neighbor interactions (with $r_{c}=a$ ), which is exactly soluble for all values of $d[5]$, and show the same LTT $\propto\left(D_{T} t\right)^{-d / 2}$ with a finite $D_{T}$, as the random DPD solid and the remaining lattice models with $r_{c}=M a$, where $M=1,2, \ldots$ In fact, the behavior of the DPD solid resembles that of Lorentz gases, where the diffusivity $D$ is also finite for all values of $d$. In fact the random DPD solid and the Lorentz gas are in several respects each others duals [7], where the VACF has the LTT $\sim t^{-1-d / 2}$.

Furthermore, there is no contradiction between the long time behavior of fluids in Eq. (12) and that of the DPD solid in Eq. (8), because $D_{T}$ in the latter case is not related to the time integral of $C_{e}(t)$, but is given by the Green-Kubo formula, involving the time correlation function $C_{Q}(t)$ of the microscopic heat current, whose time integral converges [5].

\section{ACKNOWLEDGMENTS}

The authors thank E. Ben-Naim, J. Machta, R.W. Ziff, G. Vliegenthart, P. Krapivsky, R. Brito, and P. Español for helpful discussions and correspondence. M.R. also acknowledges financial support from the Ministerio de Ciencia y Tecnología under Project No. BFM2001-0290 and FIS200401934 and the German Research Foundation (DFG) within the SFB TR6, "Physics of Colloidal Dispersions in External Fields."

\section{APPENDIX}

In this appendix we calculate the coefficient of the heat diffusion for the lattice version of the DPD model in one dimension with an interaction range $r_{c}=M a$ with $M=1,2, \ldots$ for the evolution equation (1) with the Langevin force set equal zero, and using the range function $w(r)=\frac{1}{2} \theta\left(r_{c}-r\right)$ with $[w]=r_{c}$ for the one-dimensional case. The evolution equation (1) for $r_{n m} \geqslant M a$ becomes

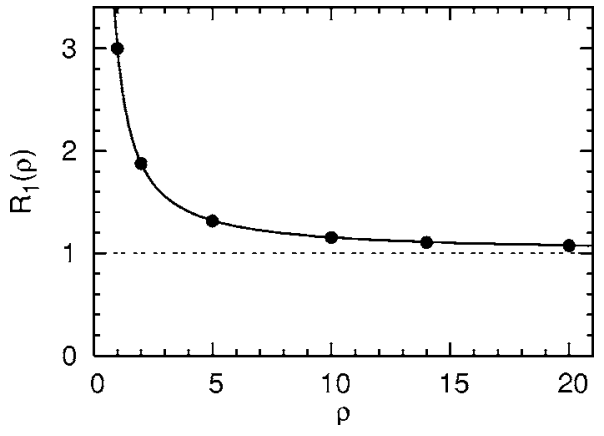

FIG. 12. A comparison of the simulations with the theoretical predictions for the heat diffusivity in the one-dimensional lattice model for $R_{1}(\rho)=D_{T}(\rho) / D_{\infty}(\rho)=(1+1 / \rho)(1+1 / 2 \rho)$ with a few simulation data. Note that $\rho$ is an integer. There is excellent agreement.

$$
\frac{\partial \epsilon\left(r_{n}, t\right)}{\partial t}=\frac{1}{2} \lambda_{o} \sum_{|m| \leqslant M}\left[\epsilon\left(r_{m}+m a, t\right)-\epsilon\left(r_{n}, t\right)\right],
$$

where $M= \pm 1$ for nearest neighbors. For large spatial scales, this discrete version of the diffusion equation is expanded to $\mathcal{O}\left(\nabla^{2}\right)$ terms included, with the result

$$
\frac{\partial \epsilon(r, t)}{\partial t} \simeq D_{T} \nabla^{2} \epsilon(r, t)
$$

with a heat diffusivity

$$
D_{T}=\frac{1}{2} \lambda_{0} a^{2} \sum_{m=1}^{M} m^{2}=\frac{1}{12} \lambda_{0} a^{2} M(M+1)(2 M+1) .
$$

It is convenient to eliminate $a$ and $M$ from this expression in favor of the interaction range $r_{c}=M a$ and of the reduced density $\rho$. Here $\rho \varpi_{d}\left(\varpi_{1}=2\right)$ is according to Eq. (2) the number of particles interacting with a given particle, which is here $2 M$. So, $\rho=M$, and the heat diffusivity becomes

$$
D_{T}(\rho)=\frac{1}{6} \rho \lambda_{0} r_{c}^{2}\left(1+\frac{1}{\rho}\right)\left(1+\frac{1}{2 \rho}\right) .
$$

In the limit of large density the heat diffusivity approaches the value $D_{\infty}(\rho)=\frac{1}{6} \rho \lambda_{0} r_{c}^{2}$. In Fig. 12 the analytic result for $R_{1}(\rho)=D_{T}(\rho) / D_{\infty}(\rho)=(1+1 / \rho)(1+1 / 2 \rho)$ is compared with computer simulations of the DPD solid in a one-dimensional lattice and shows excellent agreement. We note that for the case of nearest neighbor interactions $(\rho=1)$ the heat diffusion becomes $D_{T}(\rho=1)=\frac{1}{2} \lambda_{0} a^{2}$.
[1] B. J. Alder and T. E. Wainwright, Phys. Rev. A 1, 18 (1970).

[2] D. Levesque and W. Ashurst, Phys. Rev. Lett. 33, 277 (1974).

[3] J. J. Erpenbeck and W. W. Wood, Phys. Rev. A 26, 1648 (1982); 32, 412 (1985).

[4] M. H. Ernst, E. H. Hauge, and J. M. J. van Leeuwen, Phys. Rev. A 4, 2055 (1971); J. Stat. Phys. 15, 7 (1976).
[5] M. H. Ernst, Phys. Rev. E 71, 030101(R) (2005).

[6] J. R. Dorfman and E. G. D. Cohen, Phys. Rev. A 6, 776 (1972); 12, 292 (1975).

[7] M. Ripoll and M. H. Ernst, Phys. Rev. E 71, 041104 (2005).

[8] P. Español and P. Warren, Europhys. Lett. 30, 191 (1995).

[9] B. J. Alder and W. E. Alley, J. Stat. Phys. 19, 341 (1978); 
Physica A 121, 523 (1983).

[10] M. H. Ernst, J. Machta, J. R. Dorfman, and H. van Beijeren, J. Stat. Phys. 34, 477 (1984).

[11] J. Machta, M. H. Ernst, J. R. Dorfman, and H. van Beijeren, J. Stat. Phys. 35, 413 (1984).

[12] A. K. Harrison and R. Zwanzig, J. Stat. Phys. 42, 935 (1986).

[13] S. R. de Groot and P. Mazur, Non-equilibrium Thermodynamics (Dover, New York, 1984).

[14] P. Español and M. Serrano, Phys. Rev. E 59, 6340 (1999).

[15] M. Ripoll, Ph.D. thesis, Universidad Nacional de Educación a
Distancia, Spain, 2002 (available at www.fisfun.uned.es/ mripoll/Invest/mr-thesis.pdf).

[16] J. Quintanilla, S. Torquato, and R. M. Ziff, J. Phys. A 33, L399 (2000).

[17] C. D. Lorenz and R. M. Ziff, J. Chem. Phys. 114, 3659 (1999).

[18] M. H. J. Hagen, C. P. Lowe, and D. Frenkel, Phys. Rev. E 51, 4287 (1995).

[19] M. H. Ernst, Physica D 47, 198 (1991).

[20] M. A. van der Hoef and D. Frenkel, Phys. Rev. Lett. 66, 1591 (1991). 